\title{
From Spill to Sequestration: The Molecular Journey of Contamination via Comprehensive Multiphase NMR
}

\author{
Hussain Masoom, Denis Courtier-Murias, Ronald Soong, Werner \\ E. Maas, Michael Fey, Rajeev Kumar, Martine Monette, Henry J. \\ Stronks, Myrna J. Simpson, and André J. Simpson
}

\begin{abstract}
Version Post-print/accepted manuscript
Citation Masoom, H.; Courtier-Murias, D.; Soong, R.; Maas, W. E.; Fey, M.; (published version) Kumar, R.; Monette, M.; Stronks, H. J.; Simpson, M. J.; Simpson, A. J. Environ. Sci. Technol. 2015, 49 (24), 13983-13991.
\end{abstract}

Publisher's statement This document is the Accepted Manuscript version of a Published Work that appeared in final form in Environmental Science \& Technology, copyright (C) American Chemical Society after peer review and technical editing by the publisher. To access the final edited and published work see $10.1021 /$ acs.est.5b03251.

How to cite TSpace items

Always cite the published version, so the author(s) will receive recognition through services that track citation counts, e.g. Scopus. If you need to cite the page number of the author manuscript from TSpace because you cannot access the published version, then cite the TSpace version in addition to the published version using the permanent URI (handle) found on the record page.

This article was made openly accessible by $U$ of $T$ Faculty. Please tell us how this access benefits you. Your story matters. 


\section{From Spill to Sequestration: The Molecular Journey of}

5 Hussain Masoom ${ }^{1}$, Denis Courtier-Murias ${ }^{1}$, Ronald Soong ${ }^{1}$, Werner E. Maas ${ }^{2}$, Michael Fey ${ }^{2}$,

2. Bruker BioSpin Corp., Billerica, Massachusetts, USA, 01821-3991

3. Bruker BioSpin Canada, Milton, ON, Canada, L9T 1Y6

*Corresponding Author Information:

13 Department of Chemistry

14 University of Toronto Scarborough

151265 Military Trail

16 Toronto, Ontario.

17 Canada, M1C $1 \mathrm{~A} 4$

18 Telephone: $1+(416)-287-7547$

19 Fax : $1+(416)-287-7279$

20 Email: andre.simpson@utoronto.ca 
21 TOC ART

22

23

24

25

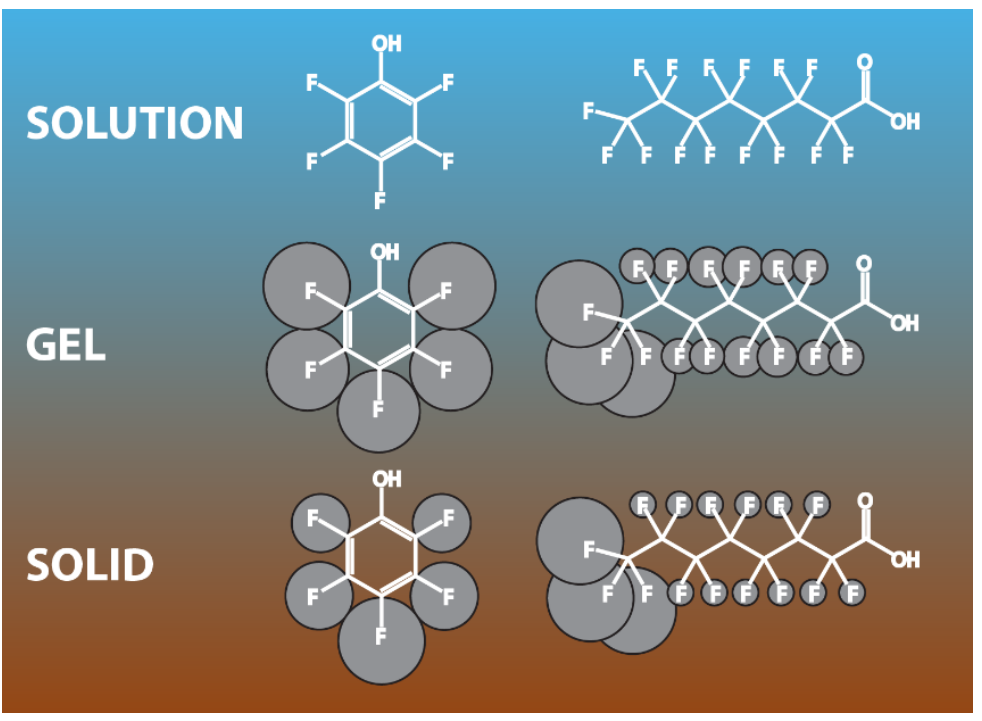



(solutions, gels, and solids) to be studied in unaltered natural samples. In this study a wide range of CMP-NMR interaction and editing-based experiments are combined to follow contaminants (pentafluorophenol (PFP) and perfluorooctanoic acid (PFOA)) from the solution state (after a spill) through the gel-state and finally into the true solid-state (sequestered) in an intact water-swollen soil. Kinetics experiments monitoring each phase illustrate PFOA rapidly transfers from solution to the solid phase while for PFP the process is slower with longer residence times in the solution and gel phase. Interaction-based experiments reveal that PFOA enters the soil via its hydrophobic tails and selectively binds to soil microbial protein. PFP sorption shows less specificity exhibiting interactions with a range of gel and solid soil components with a preference towards aromatics (mainly lignin). The results indicate that in addition to more traditional measurements such as $\mathrm{K}_{\mathrm{oc}}$, other factors including the influence of the contaminant on the soil-water interface, specific biological interactions, soil composition (content of lignin, protein, etc.) and physical accessibility/swellability of soil organic components will likely be central to better explaining and predicting the true behavior of 42 contaminants in soil. 


\section{INTRODUCTION}

$46 \quad 6$ billion Euros $(€)$ annually. ${ }^{1}$ A fundamental gap in our scientific knowledge as to how and why

47 chemicals become sequestered in the environment hampers the development of optimal

48

49

50

51

52

53

54

55

56

57

58

59

60

61

62

63

Cost estimates for the clean-up of contaminated sites in the European Union are around remediation strategies. This has led to a situation where $\sim 70 \%$ of soils from contaminated sites in Germany are being stored in vast waste disposal facilities until better clean-up technologies can be developed. ${ }^{2}$ In addition, there are important debates over the molecular fate of perfluorinated chemicals (PFCs) which are now ubiquitous in humans and the environment. ${ }^{3}$ Some scientists hypothesize the partitioning of PFCs into lipid membranes is the cause of PFC persistence in human blood whereas other groups argue the irreversible binding to human serum albumin is responsible. ${ }^{4}$ These examples demonstrate the need for novel technologies that can provide an unprecedented molecular-handle on interactions from dynamic to permanent, and across the full spectrum of states including liquid to solid in intact samples.

One major factor that impedes our understanding of contaminant-soil interactions in whole samples is the lack of analytical methods that can provide comprehensive and detailed molecular-level information on unaltered samples in their native state. Soils are very challenging to study as they are highly heterogeneous and contain a range of components in different phases. For example, organic matter dissolved in soil pores behaves as a solution, swollen organic matter is more "gel-like", whereas organic matter sorbed to mineral surfaces is closer to a true solid. 
Nuclear Magnetic Resonance (NMR) spectroscopy is a powerful tool for environmental

research and has traditionally been used to characterize soil and soil extracts. ${ }^{5}$ More recently, NMR has shown great promise in elucidating molecular interactions between soil components or between contaminants and soils or soil extracts in solution, gel, and solid phases. ${ }^{5}$ However, traditional NMR technologies, namely solution-state and solid-state NMR, focus on analysis in a single physical state. ${ }^{6}$ In 1996 an NMR technology termed High Resolution Magic Angle Spinning (HR-MAS) was introduced and allowed for swollen samples to be studied intact. ${ }^{7}$ Unfortunately, HR-MAS probes cannot handle the high power radio frequency pulses which are required for high power decoupling or cross polarization, both necessities for the study of solid components. In the case of a spill, a hydrophobic contaminant may enter the soil as a solution. As it interacts with the soil organic matter it may take on properties of a gel before being sequestered into hydrophobic pockets with more solid-like character. To fully elucidate the process of chemical sequestration it is important to be able to investigate and differentiate the molecular interactions from all phases.

Comprehensive Multiphase NMR (CMP-NMR), introduced in 2012, is a novel technology which allows for the analysis of all phases in intact unaltered samples. ${ }^{8}$ CMP-NMR incorporates a lock and full susceptibility matching (for ideal solution state line-width), magic angle spinning and pulsed field gradients (required to study the gel-state), and high power circuitry (to generate intense $B_{1}$ fields required for solid state analysis). In combination these features allow all components to be studied and differentiated in soil analysis in its whole natural swollen state thus maintaining the chemical and physical properties responsible for contaminant sorption in the environment. 
87 interactions during contaminant sequestration is demonstrated. Specifically, the fate of 88 perfluorooctanoic acid (PFOA) and pentafluorophenol (PFP) in an urban soil is tracked. Kinetic 89 transfer between the liquid, gel and solid states is monitored and the binding orientation of the

90

91

92

93

94

95

96

97

98

99

100

101

102 contaminant as well as the soil materials (protein, lignin etc.) responsible for sequestration are

identified for each phase. PFOA is commonly used in high concentration as an anti-foaming agent in the applications of agrochemicals. ${ }^{9}$ It is ubiquitous in the environment and has been found in humans and animals. ${ }^{3,10,11,12}$ PFOA has also shown to bind strongly with soil. ${ }^{13,14,15}$ Halogenated phenols like PFP have been detected in environmental systems because of their use as wood preservatives, pesticides, and herbicides. ${ }^{16}$ Perfluoronated contaminants are studied here due to their increasing environmental importance and distribution. ${ }^{3}$ In addition, the ${ }^{19} \mathrm{~F}$ nucleus is very helpful as it provides a sensitive NMR nucleus abundant in the contaminant (but not in the soil matrix) which is important for some of the experiments described here. For other contaminants that do not contain fluorine, ${ }^{2} \mathrm{H}$ labelled analogues could theoretically be used and while less sensitive should permit the study of practically any organic contaminant within a complex matrix. 
SAMPLES AND METHODS

104

105

106

107

108

109

110

111

112

113

114

115

116

117

118

119

120

121

122

123

\section{Soil and contaminant information}

The soil sample used in all experiments was obtained from Hampstead Park in Dublin, Ireland. Hampstead Park soil is a Grey Brown Podzol collected from the $A_{h}$ horizon with an organic carbon content of $6.85 \% \pm 0.42 \%$ C, $0.59 \% \pm 0.04 \% \mathrm{~N}, 4.4433 \% \pm 0.4742 \% \mathrm{Fe}, 0.0598 \% \pm$ $0.0098 \% \mathrm{Mn}$, retrieved from an open public area located within Albert College Park. This soil has a pH of 7.6. PFP (99\%) was obtained from SynQuest Laboratories (Alachua, FL, USA). PFOA (96\%) was obtained from Sigma Aldrich (Oakville, ON, Canada).

\section{Sample preparation}

All sorption experiments were performed using $30 \mathrm{mg}$ of soil in $4 \mathrm{~mm}$ zirconium NMR

rotors. For kinetics experiments, the sample was run immediately after preparation and experiments were recorded as quickly as possible. The delay time from contaminant addition to the first scan in the spectrometer was roughly two minutes. For PFP preparation, $51 \mu \mathrm{l}$ of $\mathrm{D}_{2} \mathrm{O}$ (Cambridge Isotope Laboratories, Tewksbury, MA, USA) was added to $30 \mathrm{mg}$ of pre-weighed soil in a $4 \mathrm{~mm}$ zirconium NMR rotor and mixed well using a stainless steel mixing rod, selected such that the displaced liquid is minimal and all contents remain within the rotor permitting stirring without loss. $9 \mu$ l of PFP was added after the water was added to simulate PFP entering a hydrated soil. PFOA $(7.5 \mathrm{mg})$ were dissolved in $60 \mu \mathrm{L}$ of $\mathrm{D}_{2} \mathrm{O}$ and the $\mathrm{pD}$ was adjusted to 7.0 using NaOD and a glass electrode. The deuterium ion effect was accounted for by adjusting to

7.4 on the $\mathrm{pH}$ scale. ${ }^{17}$ The $60 \mu \mathrm{L}$ solution was added into $30 \mathrm{mg}$ of soil in a $4 \mathrm{~mm}$ NMR rotor and 
124 lightly mixed. The rotor was then sealed and capped as quickly as possible to begin the kinetics 125 experiments. For the saturation transfer difference (STD) and cross polarization (CP) studies, sample 127 preparation was exactly the same as the kinetics experiments with the addition of an 128 equilibration time. Samples were prepared and stored at room temperature for 1 month before 129 STD and CP experiments were performed. This was to ensure equilibrium conditions to better 130 simulate the environment and to allow for the maximum amount of contaminant to sorb.

All experiments used in this manuscript are relatively low in sensitivity as they 132 specifically target the interactions between the contaminants and the soils and only a finite 133 number of contaminants will be interacting with any given binding site at any time. ${ }^{18}$ Many of 134 the experiments used here have been adapted from medical research to study drug binding in 135 tissue. $^{19,20,21}$ The experiments such as CP and STD selectively detect only the sub-fraction 136 interacting with the receptor (soil) and it is recommended the ligand (contaminant) is always 137 added in excess. ${ }^{20}$ For this reason the quantities used here are not reflective of what may be 138 found in the greater environment but more representative of spill conditions or agricultural 139 application. In the case of PFOA the high concentration of PFOA had little effect on the 140 aqueous-soil interface indicated by the ${ }^{1} \mathrm{H}$ NMR spectrum of the soil (see supporting Figure S1). 141 Conversely, the PFP did act like a solvent swelling the soil itself indicated by a changing ${ }^{1} \mathrm{H}$ 142 profile upon addition. This shows that the interactions may alter and may not necessarily be 143 the same as trace concentrations. The implications of this are discussed in the main text. 144 However, it also demonstrates that CMP-NMR, with the ability to study the contaminants, 145 interfaces, mechanisms, etc. is an important tool to identify which chemicals impact the soil 
146 structure/interface itself fundamental to truly understanding sorption. Considering that

147 agrochemicals are rarely applied alone and are often mixed with soil surfactants it is probable 148 that co-solvent effects are of environmental relevance and could be assessed using CMP-NMR.

NMR Spectroscopy

All NMR experiments were performed on a $500 \mathrm{MHz}$ Bruker Avance III Spectrometer magic angle gradient. All samples were spun at a rate of $6666 \mathrm{~Hz}$ and all experiments were performed at 288K. Full experimental details of the NMR experiments are provided in the 155 supporting information Section S.1.

Kinetic Curves

All kinetics curves were run in triplicate. Absolute signal integration was used to 159 calculate solution and gel experiments and the quantities of each were based on the

161 directly as the CP process is not fully quantitative. As such only relative change is reported for 162 the solid components. Estimates of the amount in the solid phase are possible through 163 difference which is discussed more in the main text. Integrals were taken using the multi164 integrate tool in AMIX (Bruker BioSpin, Rheinstetten, Germany), and relative integrals were 165 taken using the first time point as the reference spectrum. 

Epitope maps were calculated using relative signal integration through the multi168 integrate tool in AMIX. Heteronuclear saturation transfer difference (HSTD) and reverse HSTD 169 (RHSTD) epitope maps were created by comparing a saturation experiment to a standard 170 experiment without saturation and intensities were normalized to the signal that had the 171 greatest positive change in the difference spectrum (i.e. received most saturation) is denoted 172 by the $100 \% .{ }^{1} \mathrm{H}-{ }^{19} \mathrm{~F} \mathrm{CP}$ epitope maps were derived through comparison to the ${ }^{19} \mathrm{~F}$ spectrum of 173 the solid compound. 
174 RESULTS AND DISCUSSION

175 Spectral Editing of the Sub-Phases within the Soil

To isolate individual phases using CMP-NMR spectroscopy a combination of diffusion

177 filters were employed along with $\mathrm{CP}$ as a filter for true solids. These experiments have all been

178 described before by Courtier-Murias et al. ${ }^{8}$ and are only briefly described here.

179

180

181

182

183

Freely diffusing soluble species can be selected through an inverse diffusion experiment (IDE). The IDE is created by the subtraction of the diffusion edited spectrum (diffusion gradient on, contains larger components with restricted diffusion) from a reference spectrum (diffusion gradient off, contains a profile of all components) leaving just the molecules that are free to diffuse (i.e. solubilized).

Molecules with restricted diffusion can be emphasized using diffusion editing (DE). The gradient encodes the spatial position of signals at the start of the experiment and then decodes them at the end. ${ }^{22}$ The signal from molecules that diffuse and move positions are not refocused and are attenuated. Essentially the DE spectrum contains molecules that are bound and/or have restricted diffusion. To appear in diffusion editing the molecules must not move physical position, but have enough solvent induced bond dynamics to survive the relatively long delays in diffusion editing (see below for discussion of relaxation). As such, the molecules selected here in diffusion editing are going to be strongly biased towards those sorbed at the solid-water interface, which fulfills both of these conditions.

Due to the relatively long delays required for diffusion editing some molecules with fast relaxation are not observed. These signals can be recovered in a complimentary experiment 
termed Relaxation recovery Arising from Diffusion Editing (RADE). The experiment recovers the components that otherwise may be missed due to their fast relaxation if diffusion editing is used alone. ${ }^{8}$ In conventional RADE, ${ }^{1} \mathrm{H}$ is normally used for detection and true solids are not detected due to ${ }^{1} \mathrm{H}-{ }^{1} \mathrm{H}$ dipole broadening. ${ }^{8}$ However, ${ }^{19} \mathrm{~F}-{ }^{19} \mathrm{~F}$ dipole couplings are generally weaker making perfluorinated compounds easy to detect using single pulse experiments. ${ }^{23}$ Therefore, the ${ }^{19} \mathrm{~F}$ RADE will include contributions from rigid-gels, semi-solids and some true solids.

$$
\text { completely dry sample, CP-MAS is very efficient as it relies on permanent dipoles which }
$$
dominate in solid structures. However, upon swelling, water introduces local dynamics which modulate the dipolar interactions in turn reducing CP-MAS efficiency. In previous work, it has been shown that no CP-MAS signal is observed for dissolved species while mobile gels are strongly attenuated. ${ }^{8}$ In this study either the dipole between the soil and the contaminant $\left({ }^{1} \mathrm{H}\right.$ $\left.{ }^{19} \mathrm{~F}\right)$ or within the contaminant itself $\left({ }^{19} \mathrm{~F}-{ }^{13} \mathrm{C}\right)$ can be used to monitor the solid phase. In both cases, CP is most efficient if the dipole is not modulated which occurs in the true solid phase. In this study, to demonstrate the applicability of both ${ }^{1} \mathrm{H}-{ }^{19} \mathrm{~F}$ and ${ }^{19} \mathrm{~F}-{ }^{13} \mathrm{C} \mathrm{CP}$, the ${ }^{19} \mathrm{~F}-{ }^{13} \mathrm{C}$ is monitored here for the purposes of kinetics and later in the paper the more informative ${ }^{1} \mathrm{H}_{-}{ }^{19} \mathrm{~F}$ dipole is exploited in regard to binding mechanism in the solid-phase.

Figure 1 illustrates the decomposition of the spectra into the individual phases. PFP forms three distinct chemical shift bands corresponding to free (1), bound (2), and solid (3) best seen for the fluorine in the ortho position. The IDE clearly highlights the free form (Figure 1b), while the DE highlights the form with restricted diffusion (1c). The RADE identifies a range of 
material from gel through solids (Figure 1d). CP (Figure 1e) selects only the broadest components consistent with the PFP that has been sequestered into the soil to form a true solid-like phase. The use of spectral editing filters makes the assignment of the various bound states easier and less ambiguous. Unfortunately, for PFOA there are no chemical shift perturbations on binding but estimates of contributions from each phase can be performed by comparing the intensities of signal in each sub spectrum relative to the total (Figure 1a).

224 of the total ${ }^{19} \mathrm{~F}$ signal is from molecules in the dissolved state with $\sim 88 \%$ sequestered into the 225 rigid-gel, semi-solid, solid-state (Figure 1d). Conversely over half of PFOA remains in the true 226 dissolved state with $41 \%$ showing some signs of sequestration. The predicted $\mathrm{K}_{\mathrm{oc}}$ (ACD labs 227 Phys. Chem. Suite 2012) at pH 7 are 36 for PFP and 3 for PFOA which are in line with a strong 228 interaction for PFP. Interestingly the soil partition coefficient $\left(\mathrm{K}_{\mathrm{oc}}\right)$ at $\mathrm{pH} 5.5$ for PFP is predicted to be 578 while PFOA remains relatively unchanged. Therefore if lower $\mathrm{pH}$ micro environments exist within the soil these could show a strong preference towards PFP. The smaller size and roughly spherical shape of PFP may also aid in penetration into the soil. In addition, changes were noted in the ${ }^{1} \mathrm{H}$ spectrum upon the addition of PFP. PFP acts like a solvent and is capable 233 of swelling and penetrating a wider range of soil components (Figure S1). This behaviour is 234 interesting, easily observable by NMR, but not easily predictable based on the physical235 chemical properties of the molecule alone. Unfortunately the absolute percent in the true solid phase cannot be accurately 237 determined as CP cannot be quantified easily with respect to the total ${ }^{19} \mathrm{~F}$ signal. In the case of 238 PFP, as the solid components can be seen in the total spectrum, the contribution in Figure $1 \mathrm{a}$ 
239 can be used as a rough guide. It appears that a considerable fraction ( $50 \%$ based on

240 estimations from Figure 1a) is sequestered into the solid phase. Unfortunately, accurate

241 estimates are not possible for PFOA as the solid components cannot be discerned in Figure 1a.

242 However, there is $\sim 3$ times the signal-to-noise in the PFP spectrum than PFOA (Figure 1e) which

243 suggest PFP sequesters $\sim 3$ times more than PFOA. The reason behind this is not completely

244 clear but can be elucidated using other NMR techniques demonstrated in the rest of this 245 manuscript starting with individual phase kinetics.

$247 \quad$ Kinetics Between Phases

$248 \quad$ Kinetics studies are very useful to explain sorption properties including adsorption 249 mechanisms, surface properties of the adsorbent, and degree of affinity for the adsorbent. ${ }^{24}$ 250 Most commonly the concentration of sorbent left in the solution phase is used to calculate the 251 amount sorbed by difference. CMP-NMR is highly complementary as it can follow not just the 252 free contaminant but also as it penetrates into the gel and becomes sequestered into the solid 253 phase.

In this study, multiple phase kinetics were obtained in a whole soil through the use of CMP-NMR $^{8}$ and CPMG-SS ${ }^{25}$ techniques, and absolute quantification was performed in a similar

256 method as was done in Figure 1. Figure 2a plots the kinetics in the solution and gel for PFP and 257 PFOA. The solution phase kinetics curve for PFP had a small and steady decline while the 258 solution state curve for PFOA had a rapid decline and reached equilibrium quickly. In the case of 259 PFOA it is possible that the soil becomes saturated and cannot take on any more contaminant. 
260

261

262

263

264

265

266

267

268

269

270

271

272

273

274

On the other hand, the solvent like properties of PFP may aid the soil swelling allowing more molecules to penetrate.

The kinetic curve for the gel-phase PFP and PFOA (as determined by diffusion editing) both declined continuously. Essentially these are molecules that have some bond dynamics and have relatively long relaxation, but show no physical movement and will therefore be dominated by molecules bound at the soil-water interface. This signal starts at $\sim 4-5 \%$ indicating a rapid initial sorption at the interface (before the first NMR experiment) followed by a slow decline caused by slow transfer across the interface into the more rigid soil interior. For both contaminants only a small fraction $(<5 \%)$ of the contaminant was bound to the interface at any given time.

Finally, the solid state (Figure $2 b$ ), represents the fraction that has essentially become fully sequestered and taken on properties of a solid. Such soil domains are likely hydrophobic and removed from the water which itself would induce local dynamics in turn attenuating CP signal. While it is possible from Figure 1 (see discussion above) that there is $\sim 3$ times the amount of PFP in the solid phase when compared to PFOA, unfortunately direct quantification from $\mathrm{CP}$ is not possible. As such Figure 2 simply shows the relative change over time. For PFOA this is considerable, rising $~ 80 \%$ after the 2 hour window. This is correlated with a reduction in the solution fraction and demonstrates that the contaminant is crossing the soil interface and becomes sequestered into the hydrophobic interior. Interestingly the signal after the first point (10mins) is already significant (relative change 1.0) and demonstrates that more is sequestered in the first 10 mins than over the next 2 hours (relative change 1.8 after 2 hours). In turn, this demonstrates that initially the soil can rapidly take up large quantities of contaminant and this 
282

283

284

285

286

287

288

289

290

291

292

293

294

295

296

297

298

299

300

301

contaminant becomes rapidly sequestered into the solid phase. A similar trend with PFP is observed but the increase in the solid fraction and the corresponding decrease in the solution phase are less pronounced. In addition, while the solid fraction of PFP has mainly plateaued a very slight rise may still be visible after 2 hours. When considered alongside the continued decrease in the solution and gel fractions (Figure 1a,b) the data suggest the kinetics for PFP have yet to reach full equilibrium. This is likely in part due to the solvent effects of PFP which further swell the soil overtime permitting further sorption. While kinetics provides a deeper understanding of the sorption process it provides little direct evidence of the molecular-level phenomena behind the process. To address this, additional CMP-NMR experiments can be utilized to identify what soil components bind the contaminant as-well as the binding orientation of the contaminant itself.

\section{Molecular Orientation of the Contaminant}

The molecular orientation of the contaminant in both the dynamic- and solid- phase are discriminated. As both approaches use different NMR techniques the dynamic interactions will be discussed first.

\section{Orientation in the Dynamic Fraction}

A technique called heteronuclear saturation transfer difference (HSTD) was employed.

As ${ }^{1} \mathrm{H}$ atoms from the soil are saturated, the saturation perturbates through spatial dipoles and 
302 is transferred to the ${ }^{19} \mathrm{~F}$ atoms that are in closest spatial proximity to the soil. ${ }^{4,26,27,28}$ The ${ }^{19} \mathrm{~F}$

303 groups in the contaminant closest to the soil receive the most saturation, which can be 304 quantified and converted into an epitope map. Each group is normalized to the strongest 305 binding position (denoted 100\%) and interactions at other positions are given relative to this. 306 To suppress signals from solid signals, a short CPMG filter was determined empirically and was 307 included prior to acquisition. Only components that show interaction with soil will be detected 308 in HSTD while free components will be completely subtracted. Hence, the HSTD result can be 309 thought of as the average binding orientation of the dynamic fraction. This will include 310 molecules bound at the interface as well as more dynamic species partitioning with the soil. Figure 3a illustrates the results of the epitope maps calculated for PFP and PFOA for the 312 dynamic phase. The ${ }^{19} \mathrm{~F}$ position with the strongest interaction is normalized to $100 \%$ and other 313 positions were reported relative to this. PFP displayed non-specific binding since each ${ }^{19} \mathrm{~F}$ atom 314 is fairly close to $100 \%$. However, there is a very slight preference towards the $\mathrm{OH}$ indicated by 315 the strongest interaction of the ${ }^{19} \mathrm{~F}$ nuclei closest to the $\mathrm{OH}$ group. This is logical since the 316 aqueous-soil interface has been shown to be relatively polar, ${ }^{29}$ and with a pKa of 5.5 the $\mathrm{OH}$ 317 may be protonated in more acid microenvironments and could be more likely to associate with, 318 rather than repel, the soil organic matter (itself mainly anionic). Conversely with a pKa of $3.8^{30}$ 319 PFOA will be mainly negatively charged and largely repel organic matter. Indeed this is reflected 320 in its epitope map which demonstrates a bias towards the hydrophobic tail end as the point of 321 interaction. This is consistent with previous work that illustrates that ${ }^{19} \mathrm{~F}$ rich functional groups 322 play a key role for contaminant sorption to soil. ${ }^{4,26,28}$ 
The solid phase epitope map was derived from ${ }^{1} \mathrm{H}^{19} \mathrm{~F}$ CP. In this case the ${ }^{1} \mathrm{H}$

327 magnetization is passed from the soil, which acted as a source of ${ }^{1} \mathrm{H}$, to the contaminant $\left({ }^{19} \mathrm{~F}\right)$ 328 via rigid dipoles exclusive to the contaminant in the solid phase. The inherent signal from ${ }^{19} \mathrm{~F}$ is 329 supressed using phase cycling and the only way for signal to arise in the experiments is from 330 magnetization transferred from the soil protons. As such, the signal in the resulting spectrum is 331 proportional to the interaction with the soil from which an epitope map can be constructed. To 332 further support this, $\mathrm{CP}{ }^{1} \mathrm{H}^{-19} \mathrm{~F}$ build up curves were recorded for each ${ }^{19} \mathrm{~F}$ position. From these 333 curves, the heteronuclear ${ }^{1} \mathrm{H}_{-}{ }^{19} \mathrm{~F}$ dipole couplings can be extracted. Essentially both build up 334 curves and conventional ${ }^{1} \mathrm{H}_{-}{ }^{19} \mathrm{~F}$ CP produce the same binding orientations. For the sake of 335 brevity a more involved analysis of the buildup curves is presented in the supporting 336 information (supporting section S.4).

Figure $3 b$ shows the binding orientation for the molecules in the solid-state. PFP in the gel state showed little orientation preference (Figure 3a), however in the solid-phase there is a clear bias towards the most hydrophobic segment of the molecule (Figure 3b). This confirms 340 that hydrophobicity is the overall driving factor for sequestration of PFP within soil. The 341 reduced interaction at the $\mathrm{OH}$ strongly suggests the final sequestered site is removed from the 342 water interface. The solid interactions of PFOA also support this finding. However, extracting 343 information along the chain is challenging due to spectral overlap and low $\mathrm{S} / \mathrm{N}$, and therefore 344 only an average for the $\mathrm{CF}_{2}$ portion of the chain can be reported. It is clear that in the solid state 
345 the interaction further favors the hydrophobic end of the molecule and that the $\mathrm{COOH}$ group

346 has little influence. This is consistent with previous binding studies that determine the

347 halogenated functionalities in organic contaminants show the strongest preference for both soil

348 and extracted soil organic matter. ${ }^{28,}{ }^{31}$ Combined kinetics and molecular orientation studies

349 paint detailed molecular pictures as to how and why contaminants become sequestered in soil.

350 However, information regarding the exact soil components that bind the contaminants is

351 missing.

\section{Elucidating the Soil Components Responsible for Binding}

354

355

356

357

358

359

360

361

362

363

364

365

\section{Soil Components Involved in Dynamic Interactions}

To elucidate which sub-components of the soil bind the contaminant, the reverse of HSTD and CP experiments described above are employed. Reverse HSTD (RHSTD) is used to identify dynamic interactions. In this case the contaminant $\left({ }^{19} \mathrm{~F}\right)$ is saturated with magnetization which is passed onto the soil ${ }^{1} \mathrm{H}$ for detection. ${ }^{1} \mathrm{H}-{ }^{1} \mathrm{H}$ dipoles in the macromolecular soil components propagate the saturation such that the equilibrium magnetization is perturbed through the entire macromolecular network. A difference approach is used for detection and in simple terms all components that are not interacting with the contaminant cancel (due to a $180^{\circ}$ phase shift of the receiver every other scan) leaving only signals from soil components interacting with the contaminant in the final spectrum. In the case of ${ }^{1} \mathrm{H}$ detection, true solids are attenuated because they have wide spectral profiles. Therefore, the RHSTD biases interaction with dynamic soil components at the interface, in exchange, or swollen sites. 

and presents a wide range of soil components (lignin, lipids, protein, and carbohydrates) and is 368 shown in Figure 4a. This is in line with PFP having solvent like properties that can 369 indiscriminately swell all components in soil organic matter to some extent. Conversely, very 370 little if any signal is observed for PFOA even if the experiment is run for many days. This is 371 interesting as previous studies have shown with extracted soil organic matter in solution RHSTD 372 spectra can be obtained and PFOA shows a preference towards protein. ${ }^{27}$ This suggests the 373 protein fraction in extracted matter (known to be lysed from microbial cells) ${ }^{32}$ is not available at 374 the soil interface in whole samples, and reiterates the importance of studying samples in their 375 unaltered state. As well, this demonstrates the importance of physical accessibility in the 376 sorption process.

If all the data presented so far are considered, it can be seen that very little PFOA is in 378 the gel-state (Figure 1) with the majority either not interacting at all ( 60\%), or in the semi379 solid/solid-phase ( $40 \%$, Figure 1 d). As such, it seems that PFOA exhibits a more specific 380 targeted interaction. If these locations are available it will bind and become sequestered as a 381 solid, but if not PFOA will stay mainly in the solution state without swelling the soil interface 382 (see supporting section S.2). As mentioned above ${ }^{1} \mathrm{H}-{ }^{1} \mathrm{H}$ dipoles lead to spectral components for 383 solids many $\mathrm{kHz}$ wide. The lack of signal in the RHSTD suggests the components that PFOA 384 interacts with have a strong solid-like character. 
The solid phase components that are responsible for sequestration of contaminants are

identified through ${ }^{19} \mathrm{~F}-{ }^{1} \mathrm{H} \mathrm{CP}$. In this case the ${ }^{19} \mathrm{~F}$ magnetization is passed from the contaminant to the soil $\left({ }^{1} \mathrm{H}\right)$ via rigid dipoles found in the solid phase. The result is a spectrum of the soil components that bind the components in the solid phase (Figure $4 \mathrm{~b}$ ).

PFP demonstrates a markedly different spectrum than for the dynamic fraction. This spectrum is dominated by a broad aromatic resonance consistent with lignin in soil. This is in line with previous work that has shown perfluoroaromatics binding to lignin components in isolated organic matter and soil. ${ }^{27,}{ }^{33}$ It has been previously shown that lignin components are buried under the soil-water interface in a domain where water cannot penetrate soil organic matter. ${ }^{29}$ The mechanism of PFP interaction could be the following. First PFP indiscriminately swells soils such that lignin components are uncovered and once exposed shows a strong preference towards these sub-components. Previous work identifies that the overlap of the electrostatic potential density profiles between the prefluoroaromatic contaminants and the aromatic sub-units of lignin are responsible for the strong interaction in extracted organic matter. ${ }^{26}$ Ultimately, this does not rule out an interaction with other aromatic species in soil organic matter including tannin, suberin, or black carbon.

PFOA displays a strong preference towards the aliphatic components of soil. The apex of the soil component peak is centered around $0.7 \mathrm{ppm}$ for methyl ${ }^{1} \mathrm{H}$ from proteins rather than 1.3ppm which would be expected for long chain aliphatics. Indeed previous work on soil organic matter has shown that these components are from soil protein mainly derived from microbes. ${ }^{32}$ Furthermore extensive NMR based work in human blood and soil organic matter have shown that PFOA specifically binds to albumin proteins. ${ }^{4,27}$ Albumins are by design fatty acid transport 
proteins and PFOA is essentially a perfluoronated fatty acid and therefore binds to the same

410 sites, specifically Sudlow's site I, but irreversibly. ${ }^{4}$ In whole soil, it appears that the fate of PFOA

411 is the same as in extracted organic matter and that PFOA binds to the protein fraction. It is not

412 clear whether the protein fraction is within living microbial biomass or necromass. If PFOA is

413 within living microbial cells, then the microbial community could have a much more direct and

414 profound role on contaminant sequestration, fate and transformation than previously

415 anticipated. However, given the high concentrations of contaminant used here, microbial cells

416 in these experiments may be inactive because of the toxicity of PFOA. It has been previously

417 reported that in eukaryotic and bacterial cells, a relatively low concentration (milli-molar) of

418 PFOA is required to inhibit bioactivity. ${ }^{34}$ Further considerations as to the general applicability of

419 CMP-NMR are discussed in supporting section S5.

This manuscript, using PFP and PFOA as examples, has demonstrated that CMP-NMR is a

421 versatile technique to better understand and monitor contaminant sequestration at the

422 molecular-level. PFP shows more general partitioning behavior, but its ability to act as a solvent

423 and change the properties of the soil-water interface itself is hard to predict without NMR data.

424 The results indicate that the impact of the contaminant itself on the soil-water interface

425 (especially if a liquid) is an important factor to consider in predicting contaminant sorption and

426 could vary with concentration (i.e. the more contaminant the more the soil properties

427 themselves are altered) and may vary from soil to soil. Conversely, PFOA which is found to

428 selectively bind to soil microbial protein is of particular significance. The EPA reports that in its

429 ionic form PFOA can readily migrate from soil to ground water and be transported long

430 distances. ${ }^{35,36}$ The data here both support and contrast this. When binding sites are not 
431 available PFOA indeed remains in the solution phase. However, if protein binding sites are 432 available it irreversibly binds to these sites to become a solid. Considering that the microbial 433 protein content of soil varies considerably from less than $1 \%$ in acid forest soils (which are 434 dominated by fungi) to up to $50 \%$ of the carbon in grassland soils (dominated by bacteria), ${ }^{32}$ it is 435 likely the behavior of PFOA in soils will differ greatly and may in part be proportional to the 436 available protein binding sites. The EPA further report that "PFOA has a high affinity for fatty 437 acid binding protein" and that it can "interfere with fatty acid metabolism and may deregulate 438 metabolism of lipids and lipoproteins". ${ }^{35}$ As such, the impact of PFOA binding to microbial 439 protein in soil is not clear but of importance given that PFOA is used in high concentration as an 440 anti-foaming agent in the applications of agrochemicals. ${ }^{9}$ If PFOA does indeed penetrate living 441 cells and impacts some microbial species more than others then the potential to disrupt the 442 microbial diversity in soils exists. In summary the results suggest more traditional approaches 443 that predict contaminant partitioning into soils based on simple parameters such as $\mathrm{K}_{\text {oc }}$ may be 444 limited. In reality numerous and complex factors including the soil's chemistry (contents of 445 protein, lignin etc.), physical accessibility of soil components to contaminants and water, soil 446 biology (probability for contaminant to undergo specific biological interactions) and potential of 447 the contaminant itself to alter to soil-water interface likely all play important roles. 


\section{SUPPORTING INFORMATION}

449 Detailed methods on instrumental parameters for spectral editing, kinetics, and interactions;

450 Figure S1, illustrating swelling capabilities of PFP vs. PFOA as well as a discussion; Figure S2, 451 spectra used for epitope map calculations; a CP build up curve explanation; Figure S3, CP build 452 up curves; Table S1, calculated $1 / T_{H F}$ values; and a discussion on future considerations. The 453 Supporting Information is available free of charge on the ACS Publications website at...

455 ACKNOWLEDGEMENTS

456 H.M. thanks the Natural Sciences and Engineering Research Council of Canada (NSERC) for a 457 postgraduate doctoral award and the Ontario Graduate Scholarship for funding. A.J.S. thanks 458 NSERC, (Strategic and Discovery Programs), the Canada Foundation for Innovation (CFI), and the 459 Ministry of Research and Innovation (MRI) and Krembil Foundation for providing funding. A.J.S. 460 also thanks the Government of Ontario for an Early Researcher Award. 
462

463

464

465

466

467

468

469

470

471

472

473

474

475

476

477

478

479

480

1. Panagos, P.; Van Liedekerke, M.; Yigini, Y.; Montanarella, L. Contaminated sites in Europe: Review of the current situation based on data collected through a European network. J. Environ. Public Health. 2013, 2013

2. Peuke, A. D.; Rennenberg, H. Phytoremediation - Molecular biology, requirements for application, environmental protection, public attention and feasibility. EMBO Rep. 2005, 6 (6), 497-501.

3. Lindstrom, A. B.; Strynar, M. J.; Libelo, E. L. Polyfluorinated Compounds: Past, Present, and Future. Environ. Sci. Technol. 2011, 45 (19), 7954-7961.

4. D'eon, J. C.; Simpson, A. J.; Kumar, R.; Baer, A. J.; Mabury, S. A. Determining the Molecular Interactions of Perfluorinated Carboxylic Acids with Human Sera and Isolated Human Serum Albumin using Nuclear Magnetic Resonance Spectroscopy. Environ. Toxicol. Chem. 2010, 29 (8), 1678-1688.

5. Simpson, A. J.; McNally, D. J.; Simpson, M. J. NMR spectroscopy in environmental research: From molecular interactions to global processes. Prog. Nucl. Magn. Reson. Spectrosc. 2011, 58 (3-4), 97175.

6. Preston, C. M. Environmental NMR - the early years. Magn. Reson. Chem. 2015,

7. Maas, W. E.; Laukien, F. H.; Cory, D. G. Gradient, high resolution, magic angle sample spinning NMR. J. Am. Chem. Soc. 1996, 118 (51), 13085-13086.

8. Courtier-Murias, D.; Farooq, H.; Masoom, H.; Botana, A.; Soong, R.; Longstaffe, J. G.; Simpson, M. J.; Maas, W. E.; Fey, M.; Andrew, B.; Struppe, J.; Hutchins, H.; Krishnamurthy, S.; Kumar, R.; Monette, 
M.; Stronks, H. J.; Hume, A.; Simpson, A. J. Comprehensive multiphase NMR spectroscopy: Basic experimental approaches to differentiate phases in heterogeneous samples. J. Magn. Reson. 2012, $21761-76$.

9. Dinglasan, M. J. A.; Ye, Y.; Edwards, E. A.; Mabury, S. A. Fluorotelomer alcohol biodegradation yields 485

10. Kannan, K.; Corsolini, S.; Falandysz, J.; Fillmann, G.; Kumar, K. S.; Loganathan, B. G.; Mohd, M. A.;

11. Martin, J.; Whittle, D.; Muir, D.; Mabury, S. Perfluoroalkyl contaminants in a food web from lake Ontario. Environ. Sci. Technol. 2004, 38 (20), 5379-5385.

12. Kannan, K.; Corsolini, S.; Falandysz, J.; Oehme, G.; Focardi, S.; Giesy, J. P. Perfluorooctanesulfonate 493 and related fluorinated hydrocarbons in marine mammals, fishes, and birds from coasts of the Baltic and the Mediterranean Seas. Environ. Sci. Technol. 2002, 36 (15), 3210-3216.

13. Zareitalabad, P.; Siemens, J.; Hamer, M.; Amelung, W. Perfluorooctanoic acid (PFOA) and perfluorooctanesulfonic acid (PFOS) in surface waters, sediments, soils and wastewater - A review on concentrations and distribution coefficients. Chemosphere. 2013, 91 (6), 725-732. soils. Desalin. Water. Treat. 2013, 51 (40-42), 7469-7475. 
500

501

502

503

504

505

506

507

508

509

510

511

512

513

514

515

516

517

518

519

15. Milinovic, J.; Lacorte, S.; Vidal, M.; Rigol, A. Sorption behaviour of perfluoroalkyl substances in soils. Sci. Total Environ. 2015, 511 63-71.

16. Armenante, P. M.; Kafkewitz, D.; Lewandowski, G. A.; Jou, C. J. Anaerobic-aerobic treatment of halogenated phenolic compounds. Water Res. 1999, 33 (3), 681-692.

17. Krezel, A.; Bal, W. A formula for correlating pK(a) values determined in $\mathrm{D} 2 \mathrm{O}$ and $\mathrm{H} 2 \mathrm{O}$. J. Inorg. Biochem. 2004, 98 (1), 161-166.

18. Dixon, A. M.; Mai, M. A.; Larive, C. K. NMR investigation of the interactions between 4 '-fluoro-1 'acetonaphthone and the Suwannee river fulvic acid. Environ. Sci. Technol. 1999, 33 (6), 958-964.

19. Mayer, M.; Meyer, B. Characterization of ligand binding by saturation transfer difference NMR spectroscopy. Angew. Chem. Int. Ed. 1999, 38 (12), 1784-1788.

20. Mayer, M.; Meyer, B. Group epitope mapping by saturation transfer difference NMR to identify segments of a ligand in direct contact with a protein receptor. J. Am. Chem. Soc. 2001, 123 (25), 6108-6117.

21. Meyer, B.; Peters, T. NMR Spectroscopy techniques for screening and identifying ligand binding to protein receptors. Angew. Chem. Int. Ed. 2003, 42 (8), 864-890.

22. Hajduk, P. J.; Olejniczak, E. T.; Fesik, S. W. One-dimensional relaxation- and diffusion-edited NMR methods for screening compounds that bind to macromolecules. J. Am. Chem. Soc. 1997, 119 (50), $12257-12261$.

23. Karoyo, A. H.; Borisov, A. S.; Wilson, L. D.; Hazendonk, P. Formation of Host-Guest Complexes of beta-Cyclodextrin and Perfluorooctanoic Acid. J Phys Chem B. 2011, 115 (31), 9511-9527. 
24. Foo, K. Y.; Hameed, B. H. Insights into the modeling of adsorption isotherm systems. Chem. Eng. J. 2010, $156(1), 2-10$.

25. Masoom, H.; Courtier-Murias, D.; Farooq, H.; Soong, R.; Simpson, M. J.; Maas, W.; Kumar, R.; Monette, M.; Stronks, H.; Simpson, A. J. Rapid estimation of nuclear magnetic resonance experiment time in low-concentration environmental samples. Environ. Toxicol. Chem. 2013, 32 (1), 129-136.

26. Longstaffe, J. G.; Simpson, A. J. Understanding Solution-State Noncovalent Interactions between Xenobiotics and Natural Organic Matter using F-19/h-1 Heteronuclear Saturation Transfer Difference Nuclear Magnetic Resonance Spectroscopy. Environ. Toxicol. Chem. 2011, 30 (8), 17451753.

27. Longstaffe, J. G.; Simpson, M. J.; Maas, W.; Simpson, A. J. Identifying Components in Dissolved Humic Acid That Bind Organofluorine Contaminants using (1)H\{(19)F\} Reverse Heteronuclear Saturation Transfer Difference NMR Spectroscopy. Environ. Sci. Technol. 2010, 44 (14), 5476-5482.

28. Shirzadi, A.; Simpson, M. J.; Xu, Y.; Simpson, A. J. Application of saturation transfer double difference NMR to elucidate the mechanistic interactions of pesticides with humic acid. Environ. Sci. Technol. 2008, 42 (4), 1084-1090.

29. Simpson, A. J.; Kingery, W. L.; Shaw, D. R.; Spraul, M.; Humpfer, E.; Dvortsak, P. The application of H1 HR-MAS NMR spectroscopy for the study of structures and associations of organic components at the solid - Aqueous interface of a whole soil. Environ. Sci. Technol. 2001, 35 (16), 3321-3325.

30. Burns, D. C.; Ellis, D. A.; Li, H.; Mcmurdo, C. J.; Webster, E. Experimental pK(a) Determination for Perfluorooctanoic Acid (PFOA) and the Potential Impact of pK(a) Concentration Dependence on 
Laboratory-Measured Partitioning Phenomena and Environmental Modeling. Environ. Sci. Technol. 2008, 42 (24), 9283-9288.

31. Longstaffe, J. G.; Courtier-Murias, D.; Simpson, A. J. The pH-dependence of organofluorine binding domain preference in dissolved humic acid. Chemosphere. 2013, 90 (2), 270-275.

32. Simpson, A. J.; Simpson, M. J.; Smith, E.; Kelleher, B. P. Microbially derived inputs to soil organic matter: Are current estimates too low? Environ. Sci. Technol. 2007, 41 (23), 8070-8076.

33. Longstaffe, J. G.; Courtier-Murias, D.; Soong, R.; Simpson, M. J.; Maas, W. E.; Fey, M.; Hutchins, H.; Krishnamurthy, S.; Struppe, J.; Alaee, M.; Kumar, R.; Monette, M.; Stronks, H. J.; Simpson, A. J. InSitu Molecular-Level Elucidation of Organofluorine Binding Sites in a Whole Peat Soil. Environ. Sci. Technol. 2012, 46 (19), 10508-10513.

34. Mulkiewicz, E.; Jastorff, B.; Skladanowski, A. C.; Kleszczynski, K.; Stepnowski, P. Evaluation of the acute toxicity of perfluorinated carboxylic acids using eukaryotic cell lines, bacteria and enzymatic assays. Environ. Toxicol. Pharmacol. 2007, 23 (3), 279-285.

35. Emerging Contaminants - Perfluorooctane Sulfonate (PFOS) and Perfluorooctanoic Acid (PFOA); Highlights of EPA 505-F-14-001. United States Environmental Protection Agency; Washington, DC, 2014; http://www2.epa.gov/sites/production/files/201404/documents/factsheet_contaminant_pfos_pfoa_march2014.pdf.

36. Davis, K. L.; Aucoin, M. D.; Larsen, B. S.; Kaiser, M. A.; Hartten, A. S. Transport of ammonium perfluorooctanoate in environmental media near a fluoropolymer manufacturing facility. Chemosphere. 2007, 67 (10), 2011-2019. 
561

562 


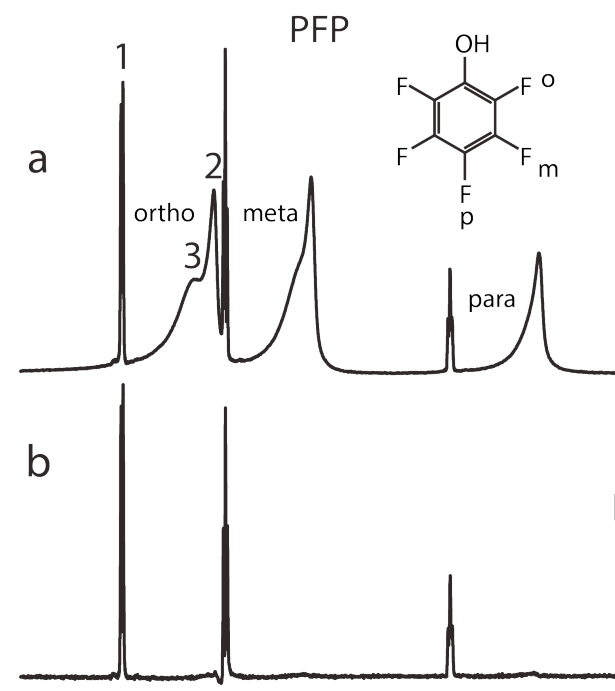

C

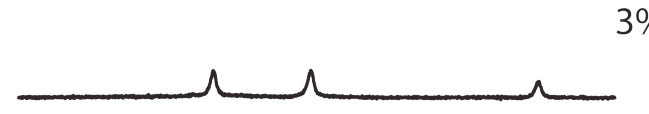

d
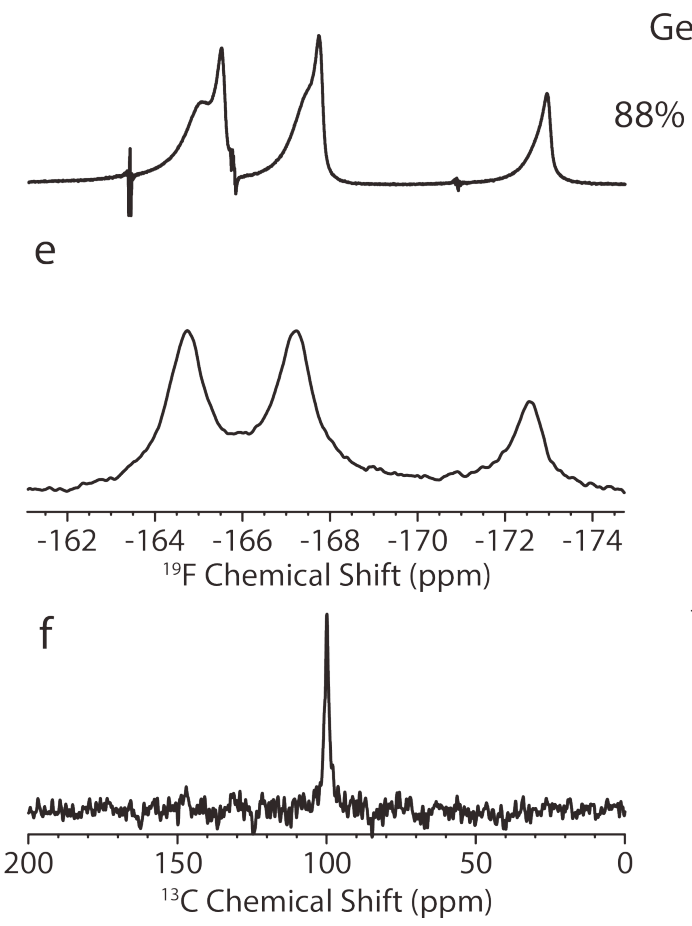

All Phases

Inverse Diffusion Editing

Solution

$$
57.5 \%
$$

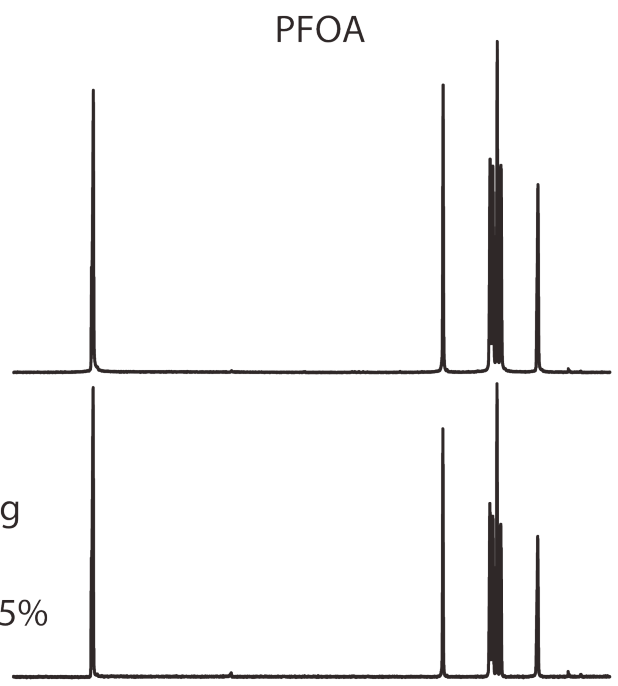

\section{Diffusion Editing}

Gel

\% $\quad 1.5 \%$

\section{RADE}

Gel/Semi-Solid/Solid

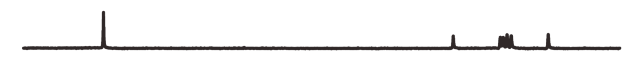

e

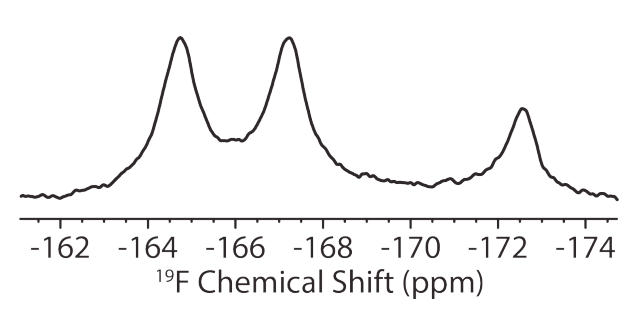

${ }^{19} \mathrm{~F}-{ }^{13} \mathrm{C}$ CPMG-SS

Solid

${ }^{1} \mathrm{H}-{ }^{19} \mathrm{~F} \mathrm{CP}$

Solid
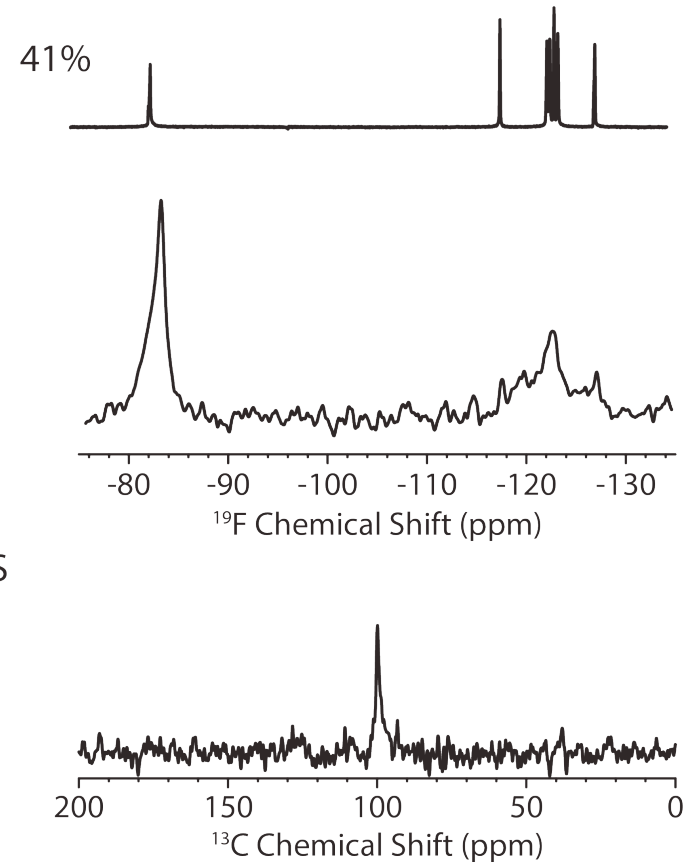
565 Figure 1: An overview of the spectral editing used in this manuscript. a) A conventional ${ }^{19} \mathrm{~F}$ 566 spectrum shows all phases. Using the ortho position as an example, solution (1), bound (2), and 567 sequestered (3) peaks can be discerned whereas this is not the case for PFOA. b) The solution 568 phase is isolated using IDE. c) Diffusion editing illustrates the small number of contaminants 569 that are at the interface of soil and water. d) The RADE accounts for any fast relaxing species 570 lost in the delays of the diffusion experiments and encompasses a range of materials from rigid 571 gels through to true solids. e) ${ }^{1} \mathrm{H}^{19} \mathrm{~F}$ CP selects for components with rigid dipoles (most solid572 like material present). PFP shows much higher signal to noise in the CP experiment indicating 573 more material sequestered into a solid-like environment. f) CP-CPMG-SS was employed to focus 574 the ${ }^{19} \mathrm{~F}-{ }^{13} \mathrm{C}$ signals into a single spike to improve sensitivity and decrease experiment time. The 575 single spike experiment permits the total amount in the solid fraction to be monitored with 576 higher temporal resolution for kinetic studies. 


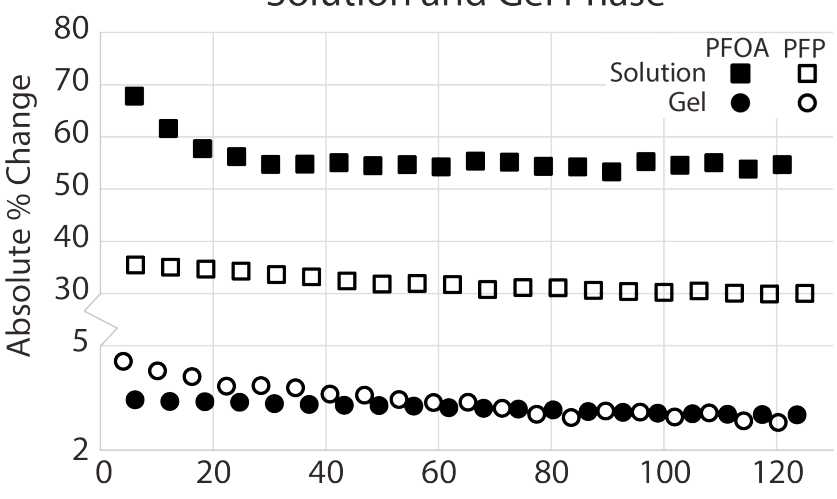

$\mathrm{b}$

Solid Phase
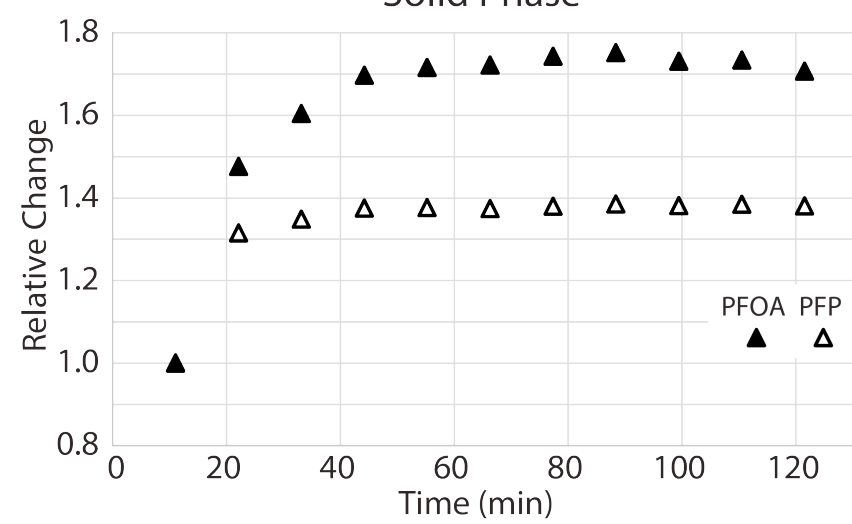

587 Figure 2: The kinetics curves for PFP and PFOA interaction in whole soil for individual phases are 588 plotted. a) Solution state curves show PFOA losing intensity quickly and reaching equilibrium 589 while PFP exhibits a steady decline. Gel phase curves show slow declines for both contaminants 590 while PFP has a stronger decline. b) Solid phase curves show a strong increase for PFOA while a 591 smaller increase exists for PFP. Standard deviations were all smaller than the width of the representative markers and were excluded for figure clarity. 
a

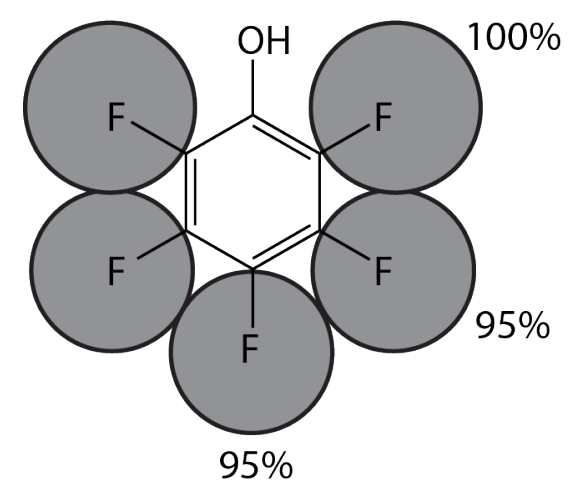

b

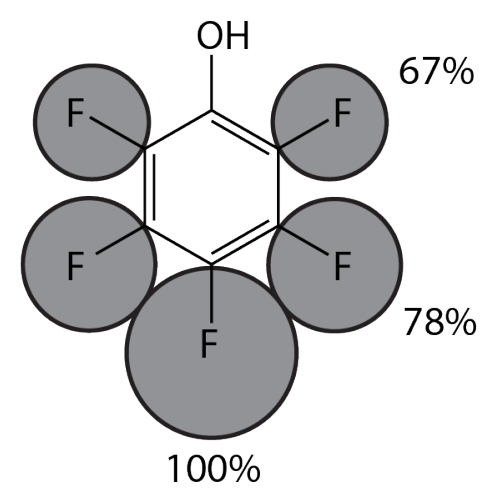

${ }^{19} \mathrm{~F}$ Gel Phase Interaction

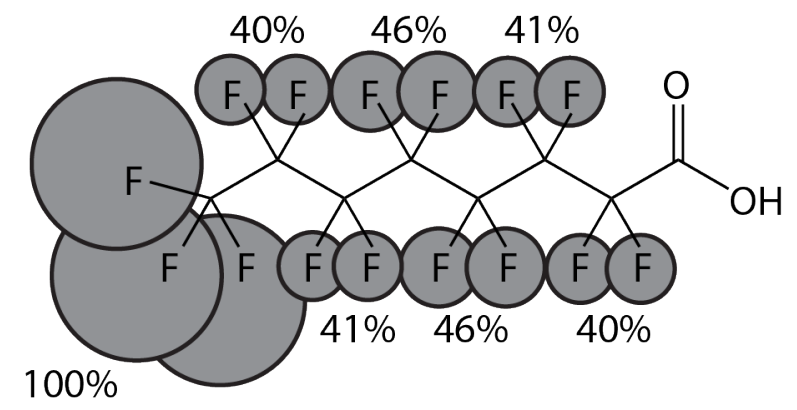

$100 \%$

${ }^{9} \mathrm{~F}$ Solid Phase Interaction

593

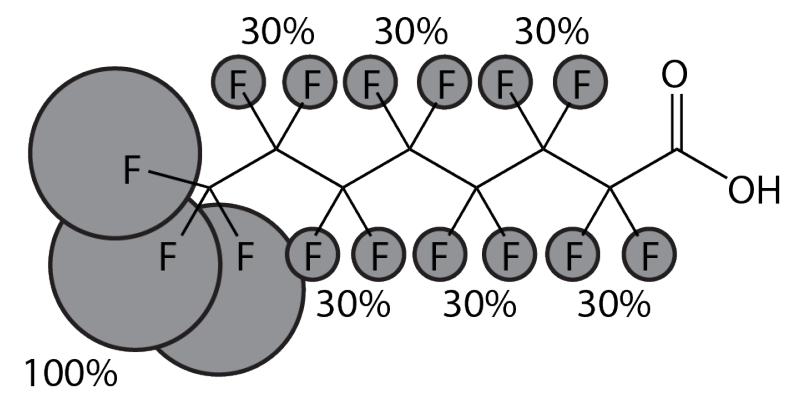

594

595

Figure 3: Epitope maps for PFP and PFOA in the gel and solid phases in whole soil were

596 calculated using HSTD and CP. For PFP, there is a discriminant shift in binding mechanism from

597 the gel to the solid phase where the $\mathrm{OH}$ group becomes less important in solid phase

598 sequestration. Conversely, the $\mathrm{CF}_{3}$ group in PFOA drives the sorption in both the dynamic and 599 solid phases. 


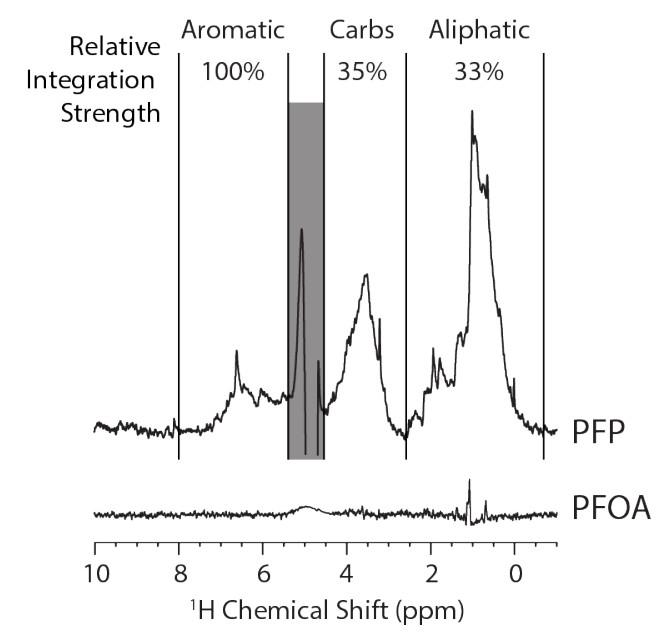

b Solid Phase Interaction Profile

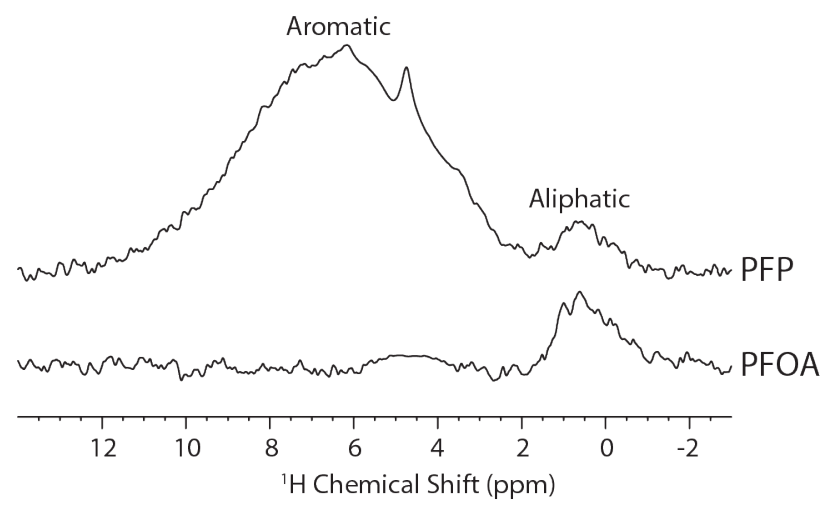

601 Figure 4: To determine which components in soil sequester PFP and PFOA, ${ }^{19} \mathrm{~F}-{ }^{1} \mathrm{H}$ RHSTD was 602 employed in the gel phase (a) while ${ }^{19} \mathrm{~F}-{ }^{1} \mathrm{H} C P$ is used in the solid phase (b). PFP shows an 603 indiscriminate profile in the gel phase showing interaction with most soil components. In the 604 solid phase however, PFP shows a strong preference to towards aromatics (lignin). PFOA shows 605 few interactions in the gel-state, but a strong preferential interaction towards proteins in the 606 solid phase. 